\title{
Empty Vehicle Redistribution with Time Windows in Autonomous Taxi Systems
}

\author{
TATIANA BABICHEVA, VEDECOM and DAVID, Université de Versailles Saint-Quentin-en-Yvelines \\ MATEJ CEBECAUER, KTH-Royal Institute of Technology \\ DOMINIQUE BARTH, DAVID, Université de Versailles Saint-Quentin-en-Yvelines \\ WILCO BURGHOUT, VEDECOM and KTH-Royal Institute of Technology \\ LEÏLA KLOUL, DAVID, Université de Versailles Saint-Quentin-en-Yvelines
}

\begin{abstract}
In this article, we investigate empty vehicle redistribution algorithms with time windows for personal rapid transit or autonomous station-based taxi services, from a passenger service perspective. We present an Index Based Redistribution Time Limited algorithm that improves upon existing algorithms by incorporating expected passenger arrivals and predicted waiting times limitations. We evaluate 17 variations of algorithms on a test case in Stockholm, Sweden. The results show that the combination of Send The Nearest and Index Based Redistribution Time Limited algorithms provides promising results for both Poisson arrivals and real demand, outperforming the other tested methods, in terms of passenger waiting time and number of passengers not served within their time windows.
\end{abstract}

CCS Concepts: • Computing methodologies $\rightarrow$ Agent/discrete models; Real-time simulation; Simulation evaluation; $•$ Applied computing $\rightarrow$ Transportation;

Additional Key Words and Phrases: Autonomous vehicles, empty vehicles redistribution, taxi management

\section{ACM Reference format:}

Tatiana Babicheva, Matej Cebecauer, Dominique Barth, Wilco Burghout, and Leïla Kloul. 2020. Empty Vehicle Redistribution with Time Windows in Autonomous Taxi Systems. ACM/IMS Trans. Data Sci. 2, 1, Article 5 (December 2020), 22 pages.

https://doi.org/10.1145/3416915

\section{INTRODUCTION}

The concept of "smart city" is being widely discussed in the literature. One of the issues of the smart city is the smart transport. The objective of this article is to design autonomous vehicles reservation and redistribution strategies allowing for a dynamic management of these vehicles.

Authors' addresses: T. Babicheva, VEDECOM, 23 bis allée des Marronniers, 78000 Versailles, France, DAVID, Université de Versailles Saint-Quentin-en-Yvelines, 45 Avenue des Etats-Unis, 78000 Versailles, France; email: tatiana.babicheva@ vedecom.fr; M. Cebecauer, KTH-Royal Institute of Technology, 100 44, Stockholm, Sweden; email: matej.cebecauer@ abe.kth.se; D. Barth and L. Kloul, DAVID, Université de Versailles Saint-Quentin-en-Yvelines, 45 Avenue des Etats-Unis, 78000 Versailles, France; emails: \{dominique.barth, leila.kloul\}@uvsq.fr; W. Burghout, VEDECOM, 23 bis allée des Marronniers, 78000 Versailles, France, KTH-Royal Institute of Technology, 100 44, Stockholm, Sweden; email: wilco.burghout@ abe.kth.se.

Permission to make digital or hard copies of all or part of this work for personal or classroom use is granted without fee provided that copies are not made or distributed for profit or commercial advantage and that copies bear this notice and the full citation on the first page. Copyrights for components of this work owned by others than the author(s) must be honored. Abstracting with credit is permitted. To copy otherwise, or republish, to post on servers or to redistribute to lists, requires prior specific permission and/or a fee. Request permissions from permissions@acm.org.

(c) 2020 Copyright held by the owner/author(s). Publication rights licensed to ACM.

2577-3224/2020/12-ART5 \$15.00

https://doi.org/10.1145/3416915 
Personal Rapid Transit (PRT) [17], also referred to as podcars, is an on-demand transportation system that uses a set of small driverless (and often electric) vehicles to transport people on demand from point to point (or station to station). Research within PRT [10] has traditionally assumed guideways (elevated or not) exclusively used by the PRT vehicles. Due to the construction cost of such guideways, as well as the rigidity of the available routes they provide, the world has seen only a handful of implementations of PRT services (e.g., Morgantown PRT and Heathrow) [19, 24].

Given the recent rapid development of self-driving (also called autonomous) vehicles, the need for guideways is disappearing, as autonomous taxis will be able to use existing roadways and thus provide door-to-door public transport services. In addition, the current trend towards "Transport as a Service" [18] or "Mobility as a Service" [29], where cars (and bicycles, etc.) are shared using increasingly convenient online platforms, revolutionises the market for transportation of people and provides new ways to match planned and unplanned travel demands with transport services. Thus, one can consider PRT and autonomous taxi services to be equivalent. An essential prerequisite for any such service is the efficient operation of the vehicle fleet, maximising the service to the passengers while minimising the cost to the operator(s).

One component of taxi system management is the way in which vehicles are assigned to passengers and how they are redistributed in the network to optimally provide this service. In both PRT and autonomous taxi systems, the service is demand responsive, where most passengers request an immediate service. The demand is known (possibly partially) or predicted based on historical data by the global management system that interacts with all autonomous taxis or shuttles. In addition, the passenger demand is often asymmetric across the network and over time, causing imbalance between vehicle supply and passenger demand in the system. Therefore, it is necessary to redistribute the empty vehicles based on demand and supply attributes to trim the system. This system should allow customers to reserve a self-driving vehicle, either in advance or on the fly (for a trip as soon as possible).

The objective of this work is to provide methods of empty vehicle management for autonomous taxi management systems, taking into account the time windows. The proposed methods have to answer two main questions: which vehicle to assign to which customer and how to redistribute empty vehicles. These two problems are NP hard, and only heuristic-based solutions are possible.

The remainder of the article is organised as follows. In Section 2, we provide an overview of previous research about user preferences in transportation problems and the empty vehicle redistribution problem. Section 3 describes the model and empty vehicle redistribution optimisation problem. Section 4 provides a description of the Index Based Redistribution (IBR) algorithm, the evaluation of the algorithms we propose and that in the literature on sample line network, and finishes with new Index Based Redistribution Time Limited (IBRTL) and Heuristic Nearest Neighbours Time Limited (HNNTL) variations of IBR and Heuristic Nearest Neighbours (HNN) algorithms. In Section 5, we describe the computational experiments design and compare the algorithms (and combinations of algorithms) on a proposed simplified autonomous taxi network of the case study of Stockholm, Sweden. We provide the analysis of time window size to the quality of service from the passengers' perspective, the comparison of problems with and without time windows under different empty vehicle redistribution algorithms, and the simulation results on real passenger demand data. To conclude, Section 6 discusses limitations of this work and future research directions.

\section{RELATED WORK}

People in developed countries spend many hours of their life traveling to work and to home, and waiting for taxis and trains. Thus it would be profitable to lessen this time. In transport 
investments, the concept of "time value" is widely used. The time of consumer becomes one of goods to buy.

Until the mid-1990s, the time value was considered to be linear (equal to some percentage of wage rate [5, 26, 28]) or considered as one of possible passenger activities [12, 14, 21, 27]. Work in the mid-1990s confirmed and quantified non-linearities in time value and revealed a group of nonlinearities. Subsequent studies with a more complex analysis of the responses about stated choice in general confirmed and corroborated these findings. In 2001, Hultkrantz and Mortazavi [20] used the Swedish data and found non-linearities of time value. Based on the data collected through two surveys carried out in 2007 and 2008, in 2014 Börjesson and Eliasson [7] clearly showed the nonlinearity of the value of travel time changes.

One important issue to consider is to minimize passengers waiting time and thus to maximize the passengers' utility. One of the problems corresponding to this issue is the empty vehicle redistribution problem which can be formulated as a dynamic version of the Vehicle Routing Problem with Time Windows [13]. However, the Vehicle Routing Problem with Time Windows can be reduced to the Traveling Salesman Problem and is therefore NP hard. As a result, most methodologies in the literature use heuristics rather than exact methods to find acceptable solutions within reasonable time. The methods for empty vehicle redistribution can be divided into two main groups corresponding to two different problems. The first group, reactive redistribution strategies, in which the call for a vehicle is made at the moment of passenger arrival, includes methods of reaction to demand. In such algorithms, an empty vehicle is to be sent in the interest of particular passengers (the one with maximal waiting time, the one closest to the vehicle, etc). The second group, proactive redistribution strategies (sending empty vehicles in anticipation of predicted demand in the near future), is the empty vehicle redistribution in the interest of the entire system. In the literature, these methods are also respectively referred to as "the demand mode of operation" and "the schedule mode of operation," corresponding to different demand levels of the system, and the schedule mode is used during time periods where there may be knowledge or strong heuristics about the future passenger demand. In some PRT systems [19], both types of empty vehicle redistribution are used. The dispatching technics are also widely used in mobile transportation, logistics, and so forth. However, in these areas, the character of these strategies is different. Mostly they have clustering methods as a basis, sometimes together with machine learning methods, such as contextual cooperative reinforcement learning [25] or deep reinforcement learning [22]. The main difference between dispatching technics in these areas and the classical empty vehicle redistribution is the nature of trips distribution (courier services have a limited set of origins, and ambulance services have a limited set of destinations). The methods for dispatching over a long horizon can take into account a more exact nature and set more objectives, such as an optimising long-term global efficiency, including large-scale platforms [30]. However, these methods require more exact spatio-temporal analysis and thus, in contrast with classical EVR strategies, can provide less efficiency in case of unpredicted changes.

Based on the literature research, the methods for empty vehicle redistribution can be divided into the following categories:

\section{- Reactive (Sending) Algorithms}

Basic allocation (BA): If there are waiting passengers and empty vehicles at the same station, empty vehicles will be assigned to the longest waiting passengers (no redistribution of empty vehicles to other stations). Mostly this strategy is a baseline strategy and is used to evaluate the real redistribution methods.

Simple Nearest Neighbours (SNN): Reallocation of the nearest empty vehicles to serve the longest waiting passengers. This type of algorithm is used in Andréasson [1], 
Fatnassi et al. [16], Fagnant and Kockelman [15], and Lees-Miller [24]. In this article, we use our implementation of the SNN algorithms as described by these authors.

Heuristic Nearest Neighbours: Reallocation of the nearest empty vehicles to serve the longest waiting passengers, taking into account the time it takes for vehicles to get to the passengers. This method attempts to improve on SNN by taking into account the time it takes for a vehicle to move towards a waiting passenger. The difference between the SNN and HNN methods can be significant. Consider a network in a form of a long edge with one empty vehicle at one end of this edge and two passengers waiting, one at each end of the edge. If the SNN algorithm is applied, the vehicle will move towards the currently longest waiting passenger, regardless of how long it takes to get to this passenger. However, if the HNN is applied, the vehicle will move to the other end of the edge because at the moment of its arrival the waiting time of the farthest passenger will be the largest. This method is mentioned by Kek et al. [23], and Bell and Wong [6].

Send The Nearest (STN): Reallocation of nearest empty vehicles is based on the nearest pairs between the available vehicles and waiting passengers. The stations with waiting passengers are to be ranged based on the time from the nearest available vehicle to the station. This method is described by Babicheva et al. [2, 3].

\section{- Proactive (Redistribution) Algorithms}

Index Based Redistribution: The redistribution of empty vehicles is based on maximal station index. The idea of the station index is related to the "virtual station status" described by Kek et al. [23], but it uses a different method for index calculation (the authors focus on the number of vehicles and do not consider the passenger waiting times). This method is described by Babicheva et al. [2, 3] and in Section 4.1.1.

Surplus/Deficit vehicle Redistribution (SDR): The redistribution of empty vehicles from the station with the maximal vehicle surplus to the station with the maximal vehicle deficit. This method was used by Andréasson [1]. The vehicle surplus/deficit is defined for each station as the number of vehicles available (currently or within a defined time horizon) minus the number of passengers who are waiting (currently or within the defined time horizon).

However, none of the existing methods is the best. In different networks or under different passengers and vehicle positions, each of methods (with exception of the BA method) can be the best suitable. Babicheva et al. [2-4] showed that combinations of algorithms show better performance for the empty vehicle redistributions problem without time windows. This explains why in this article we focus on both reactive and proactive redistribution strategies. We also propose new methods of empty vehicle redistribution (HNNTL and IBRTL). These algorithms aim to diminish the idle run to stations where passengers are not ready to wait, as well as to diminish the computational complexity in big networks.

\section{MODELLING THE EMPTY VEHICLE REDISTRIBUTION WITH TIME WINDOWS}

To formalise the proposed methods, we provide the following model of the taxi system.

\subsection{The Model Description}

The time in the model, noted $t$, is discrete with step equal to 1 second. 
3.1.1 The Network Representation. Let us have a city street network primal representation as a directed weighted graph $G=G(V, A)$, where

every vertex $v \in V$ is a road intersection or a taxi station in a street. The road intersections have generally the degree 3 or more. The taxi stops in the streets are modelled by vertices of degree 2 . We consider the system with station-to-station taxis, and thus every origin and destination points of passengers are vertices of the graph.

every arc $a \in A$ is defined as $a=\left(\right.$ init $_{a}$, ter $_{a}$, time $\left._{a}(t)\right)$, where init $_{a}$ and ter $_{a}$ are the initial and terminal points of $\operatorname{arc} a$. The times to pass the arcs are time-dependent functions, with constant expectancies for every day and day period.

3.1.2 The Demand Representation. Every passenger group $p \in P$ (from this point, we refer to it as "client") that arrived to the system can be characterised by the following parameters:

Number of passengers $N_{p}$ : Passengers who arrived together should travel together. We consider that size of the passenger group is smaller or equal to the capacity of the vehicle.

Origin $v_{p}^{o} \in V$ is the pick-up point of client $p$.

Destination $v_{p}^{d} \in V$ is the drop-off point of client $p$.

Time window is represented by ordered pair of times $\left(t_{p}^{0}, t_{p}^{1}\right)$, where $t_{p}^{0}$ and $t_{p}^{1}$ are the minimal and the maximal time of picking up, respectively. To have the possibility to serve a client, the constraint $t_{p}^{0}<t_{p}^{1}$ has to be satisfied. In this article, we consider time window size $T W_{p}=t_{p}^{1}-t_{p}^{0}$ to be constant.

Passenger generation $t_{p}^{a p p}$ : Passengers are generated according to a predefined stochastic or deterministic process. For the remainder of this article, we assume a stationary Poisson process [8] for the random appearance of passengers in the system, which is the moment when the client calls the taxi and from this moment the system knows that this client exists. In case of predefined information, in Section 5.4 the actual demand and arrival pattern for that specific day in the Stockholm data set is used.

\subsection{The Problem Setting}

The inputs of the model are as follows:

The graph $G(V, A)$ that represents the city network, or the OD matrix with minimal travel times.

The predefined demands in the form of clients with all of the attributes such as number of passengers, origin, destination and time window. Note that the passenger is recognised by the system at the moment of the taxi call $t_{p}^{a p p}$.

The vehicle set with its start positions.

The output of the model is the set of all movements done by the vehicles (with information of empty or non-empty trips), which leads also to all client trip information.

The expected quality of the system is the synthesis of expected quality from both client and service perspectives. The expected quality from the client perspective can be described as function of the waiting time only (time from the desired departure moment until the real departure). The expected quality from the service perspective can be described as function of total number and duration of empty and non-empty trips. 
The objectives in this article can be formulated such as follows:

(a) To maximize the number of clients served within their time windows

$$
\max \sum_{p \in P} F_{p}
$$

where $F_{p}=1$, if client $p$ was served within its time constraint, and $F_{p}=0$, if it quitted the system without being served.

(b) To minimize the average and maximal waiting times of served clients

$$
\begin{array}{r}
\min \frac{\sum_{p \in P, F_{p}=1}\left(t_{p}^{\text {dep }}-t_{p}^{0}\right)}{\left|p \in P, F_{p}=1\right|}, \\
\min \max _{p \in P, F_{p}=1}\left(t_{p}^{\text {dep }}-t_{p}^{0}\right),
\end{array}
$$

where $t_{p}^{d e p}$ is real departure time of client $p$.

Different algorithms can show optimums for different objective functions. The optimum obtained in terms of maximal waiting time does not have to lead to minimal average waiting time. The following simplification of real-life taxi service is considered. The client does not need immediate report from the taxi service. The client is considered to be satisfied if it is taken within its constraints and is considered to be not satisfied if any of the constraints are not respected. The client will quit the system at the end of its time window even if its taxi is already approaching.

\section{EMPTY VEHICLE REDISTRIBUTION ALGORITHMS}

In this section, first we discuss the IBR algorithm and show that the HNN algorithm can be described as a highly simplified version of IBR. The next section discusses the HNNTL and IBRTL methods, and the following section shows the mathematical evaluation of different algorithms on a simple linear network.

\subsection{The IBR Algorithm}

In this section, we discuss the main lines of the IBR algorithm proposed by Babicheva et al. [3]. This method incorporates the expected client waiting time into a numeric index, thereby making it responsive to the expected waiting times and the (expected) length of client queues.

4.1.1 The Station Index Calculation. The client's acceptable waiting time is upper bounded by some value and can be different for different clients. In addition, the client preferences for waiting times are non-linear. To take this fact into account, and to simplify calculating potential client heuristics (e.g., to introduce risk aversion), a (dis)utility $u_{p}\left(t_{p}^{d e p}-t_{p}^{0}\right)$ as a function of client waiting time is introduced. For the remainder of this article, we choose therefore the risk-loving function with exponential shape $u_{p}(t) \equiv u(t)=\exp (t)-1$ as showing the best performance after the utility function comparison.

For every station, we introduce the "station index," which is calculated as a measure of expected maximum client disutility at the time of pick-up with the nearest vehicle, including the time it takes for the vehicle to arrive at the station. The station index calculation is based on clients already arrived and on "virtual" clients who will arrive in certain time periods. Let $P_{\text {arr }>0}\left(t_{1}, t_{1}+\tau\right)$ be the probability of at least one client arrival during the time period $\left(t_{1}, t_{1}+\tau\right)$.

For every station $s \in V$, we determine the nearest available vehicle $v_{s}^{\text {nearest }}$ (given its current or next trip). Given the current time moment $t_{1}$, we calculate that $v_{s}^{\text {nearest }}$ will arrive to the station at the time moment $t_{2}$. The calculation of $v_{s}^{\text {nearest }}$ in graph representation is based on the nearest time in this graph. In OD minimal time representation, it is the time to reach the current destination of the vehicle plus the time from the current destination to station $s$. 
For every station $s$, the "client surplus" $\left(C S_{s}\right)$ is calculated as the total number of clients currently at this station minus the total number of vehicles moving to this station to take clients.

Suppose the current time from the start of simulation is $t_{1}$ and it was calculated that the nearest vehicle will arrive to the station at the time moment $t_{2}$. Thus, for station $s$, the "station index" $I_{s}$ is calculated as follows:

If $C S_{s}>0$ (vehicle deficit): Let the maximum waiting time among the clients at station $s$ be $T_{s}=\max _{p \in P_{s}} t_{p}$, where $t_{p}$ is the current waiting time of passenger $p$. The station index $I_{s}$ is defined as $u\left(t_{2}-t_{1}+T_{s}\right)$. In other words, the station index is defined as the disutility of the longest waiting client at the station, at the time of its predicted departure.

If $C S_{s}=0$ (balanced): The station index $I_{s}$ is equal to the maximal expected disutility of the first arriving client:

$$
I_{S}=\max _{t_{1}<\tau<t_{2}}\left[P_{s, \operatorname{arr}>0}\left(t_{1}, \tau\right) u\left(t_{2}-\tau\right)\right] .
$$

If $C S_{s}=-X$ (vehicle surplus of $X$ vehicles): The time expectancy $T_{s, X}$ of $X$ clients arriving is calculated from the given type of demand distribution. If $t_{1}+T_{s, X} \geqslant t_{2}$, station index $I_{s}=0$, and otherwise it is calculated as in the $C S_{s}=0$ (balanced) case, changing the start time from $t_{1}$ to $t_{1}+T_{s, X}$ :

$$
I_{s}=\max _{t_{1}+T_{s_{X}}<\tau<t_{2}}\left[P_{s, \text { arr }>0}\left(t_{1}+T_{X}, \tau\right) u\left(t_{2}-\tau\right)\right] .
$$

In other words, in case of non-positive client surplus, the index equals the probable disutility of the first arriving client at the time of its departure.

To generalize the three preceding cases, the station index is a measure of expected maximum client disutility at the time of pick-up, including the time it takes for the nearest vehicle to arrive at the station.

4.1.2 The Empty Vehicle IBR Algorithm. The IBR is based on the station indexes described earlier.

Given the station $s$ with maximal station index, if there is an available vehicle $v_{s}^{\text {nearest }}$ at station $s$, assign it to the longest waiting client, if any. If there are no waiting clients (yet), reserve $v_{s}^{\text {nearest }}$ for the next arriving client.

If there is no available vehicle at station $s$, vehicle $v_{s}^{\text {nearest }}$ will be chosen as nearest available vehicle at other stations or moving in the network. There are three cases now:

- Vehicle $v_{s}^{\text {nearest }}$ is idle at its station. Then vehicle $v_{s}^{\text {nearest }}$ will move to station $s$ to serve a client.

- If vehicle $v_{s}^{\text {nearest }}$ is moving and its destination is station $s$, then $v_{s}^{\text {nearest }}$ is reserved for serving the longest waiting client immediately after reaching $s$.

- If vehicle $v_{s}^{\text {nearest }}$ is now in a trip to another station, it is booked to make its next trip to station $s$.

4.1.3 The HNN Algorithm. The HNN can be described as highly simplified IBR, without taking into account the expected client arrivals and with the linear utility function $u(t)=t$. Thus, the HNN redistribution can be described as the IBR changing the index $I_{s}$ to $T_{s}^{m}$-the maximal waiting time within clients at station $s$ at the arrival moment of the nearest vehicle.

\subsection{The HNNTL and IBRTL Algorithms}

The empty vehicle redistribution problem with time windows differs from the classical empty vehicle redistribution problem in one main aspect considered in this article: for every client, there 
is a predefined waiting time window, and when the client reaches its time window limit, it will quit the system. Thus, in the optimisation problem, there may not be a reason to send a vehicle to the passenger who will quit the system before the vehicle arrives to the station.

Taking into account these considerations of a client's time windows, the IBR and HNN algorithms were updated to not consider in the optimisation problem clients who cannot be served within the time windows. We refer to the updated algorithms as IBRTL and HNNTL.

Therefore, the calculation of the maximal waiting client time at the moment of nearest vehicle $\operatorname{arrival} T_{s}^{m}$ for the station $s$ for the HNNTL method is changed as follows: for every station $s$ in the system, the vehicle $v_{s}^{\text {nearest }}$ is found. Suppose that the current time from the start of simulation is $t_{1}$ and the calculated nearest vehicle will come to station $s$ at the time moment $t_{2}$.

Thus, the index $T_{s}^{m}$ can be calculated as follows:

$$
T_{s}^{m}=\max _{p \in s, t_{2} \leqslant t_{p}^{1}}\left(t_{p}^{0}+t_{2}-t_{1}\right)
$$

and it represents the maximal waiting time within all clients at the station $s$ within the time window at the moment of the nearest vehicle arriving. The $T_{s}^{m}$ calculation is described in detail in Algorithm 1.

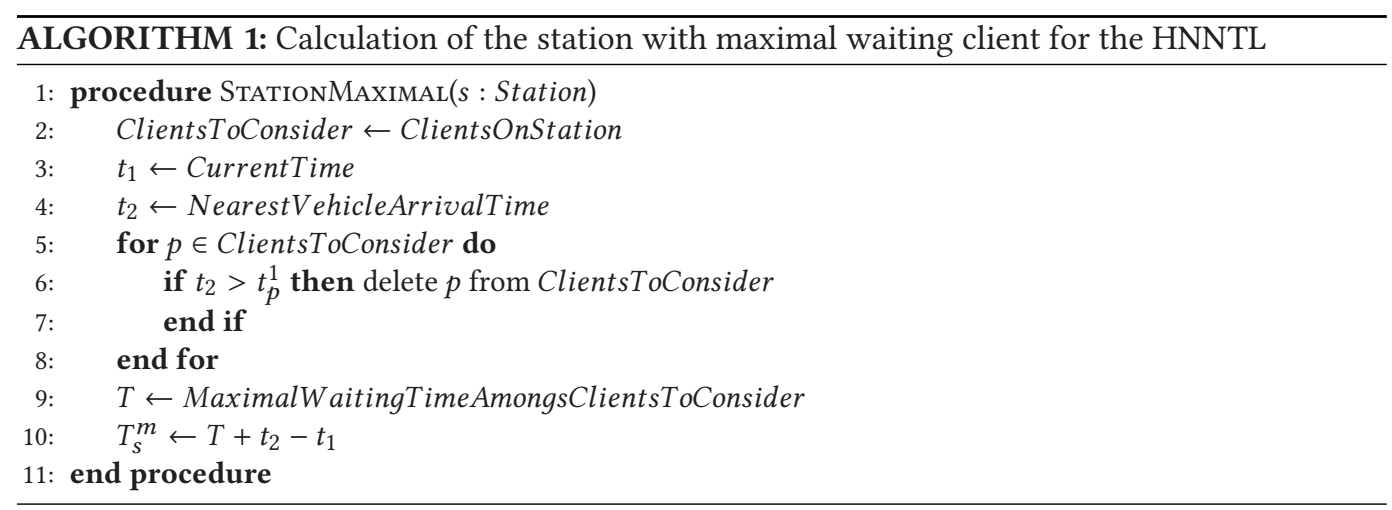

The calculation of the station $s$ index $I_{s}$ for the IBRTL method is changed as described in the following. For every station, only passengers, such that the current time of the passenger plus the time needed to reach this station by the nearest vehicle is less than or equal to the time limit, are considered. Thus, the current list of passengers in these stations is updated to not take into account the passengers who cannot be served within their time window. The index $I_{s}$ calculation is described in detail in Algorithm 2.

In this article, the comparison of the IBR and HNN methods with IBRTL and HNNTL will be provided in the case studies with time windows. The common IBR and HNN methods can be obtained from IBRTL and HNNTL when considering the infinite time windows, so the values of the objective functions of these methods will differ.

\subsection{The Empty Vehicle Redistribution Algorithms Evaluation}

In this section, we evaluate different redistribution algorithms mathematically on a simple linear network to see if any of the methods outperform the others in terms of average and maximum waiting times.

Let us consider a single line network with two stations and two vehicles (Figure 1). Each station has one client waiting for a vehicle. The driving time between the two stations is 5 minutes. We will now evaluate four cases with different locations of vehicles. 


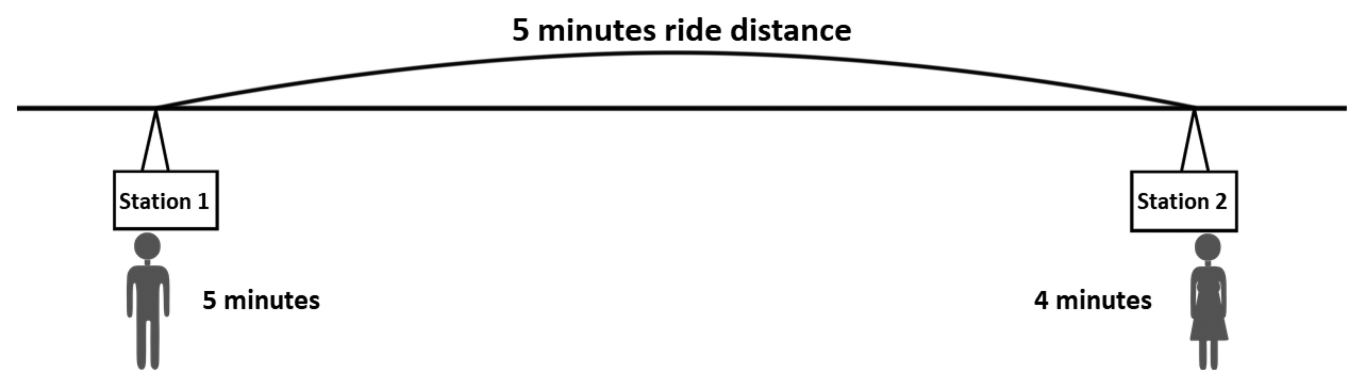

Fig. 1. The simple line network.

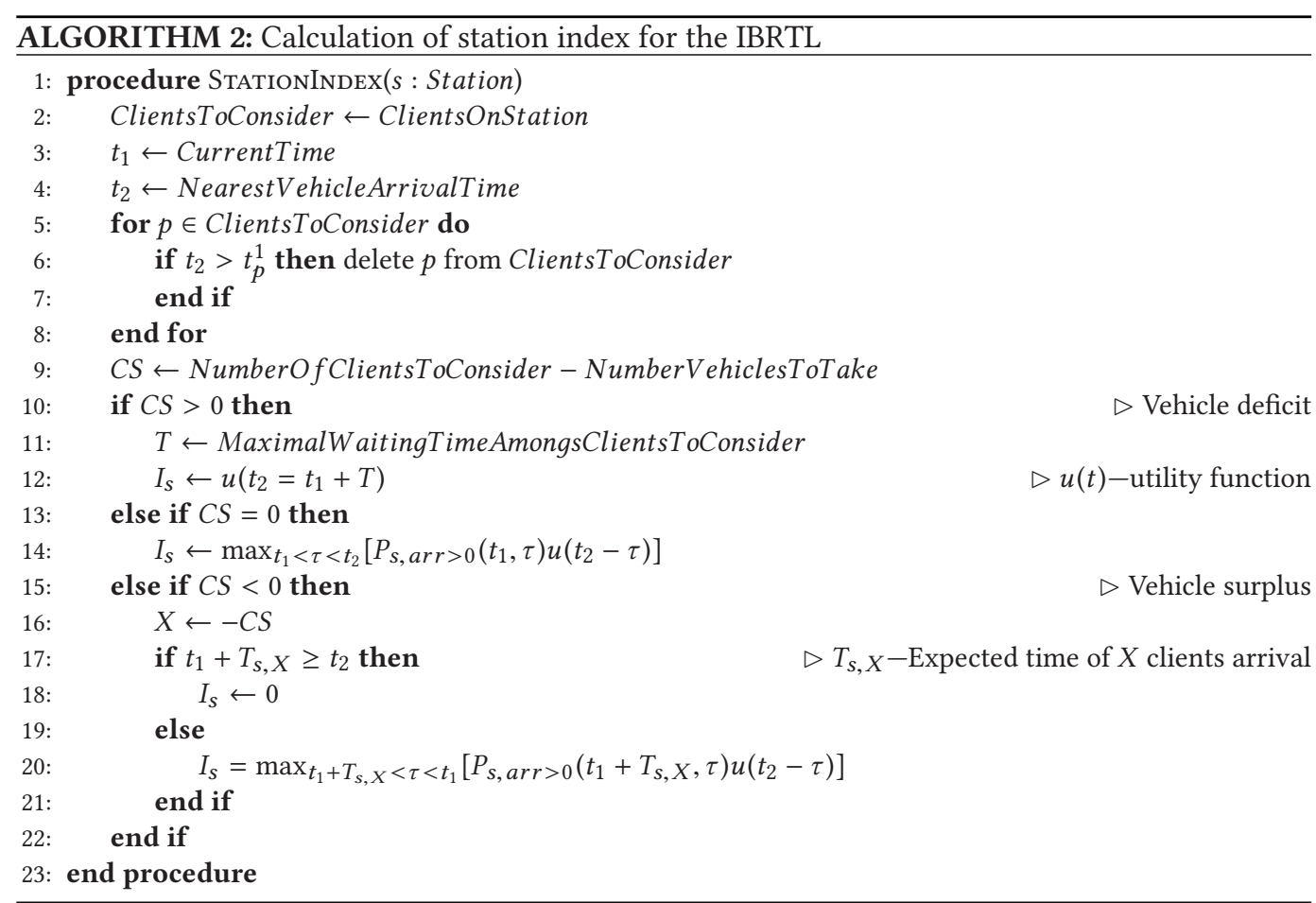

For such a network, the HNN method shows the same results as the IBR method because this case study does not consider predicted passengers. The Surplus/Deficit redistribution can be applied only when vehicles are placed at the stations, so this method is not applicable to this case study.

We provide the comparison of the SNN, IBR and STN methods for the problem with time windows. The three algorithms were evaluated and compared to see which algorithm produces the best maximum waiting time and the best average waiting time for the clients by Babicheva et al. [4].

We provide the analysis of four cases:

Case 1: The first vehicle is located at a 3-minute ride time from station 1 and the second vehicle is located between station 1 and station 2, with a ride time of 1 minute to station 1 and 4 minutes to station 2 . 
Table 1. Average and Maximal Waiting Times for the Problem Without Time Windows, in Minutes

\begin{tabular}{ccccccccc}
\hline & \multicolumn{2}{c}{ Case 1 } & \multicolumn{2}{c}{ Case 2 } & \multicolumn{2}{c}{ Case 3 } & \multicolumn{2}{c}{ Case 4 } \\
\hline & Avg & Max & Avg & Max & Avg & Max & Avg & Max \\
IBR & $\mathbf{8}$ & $\mathbf{8}$ & 13.5 & 16 & 11.5 & 15 & $\mathbf{7 . 5}$ & $\mathbf{9}$ \\
SNN & 9 & 12 & 13.5 & 16 & $\mathbf{7 . 5}$ & $\mathbf{9}$ & $\mathbf{7 . 5}$ & $\mathbf{9}$ \\
STN & 9 & 12 & $\mathbf{8 . 5}$ & $\mathbf{1 2}$ & $\mathbf{7 . 5}$ & $\mathbf{9}$ & 8.5 & 12 \\
\hline
\end{tabular}

Table 2. Number of Not Served Passengers for the Problem with Time Windows

\begin{tabular}{lcccc}
\hline & Case 1 & Case 2 & Case 3 & Case 4 \\
IBR & $\mathbf{0}$ & 2 & 1 & $\mathbf{0}$ \\
SNN & 1 & 2 & $\mathbf{0}$ & $\mathbf{0}$ \\
STN & 1 & $\mathbf{1}$ & $\mathbf{0}$ & 1 \\
\hline
\end{tabular}

Case 2: The second vehicle is located at a 7 -minute ride time from station 1, and the first vehicle is located at a 1-minute ride time from station 1.

Case 3: The first vehicle is located between station 1 and station 2, with a ride time of 1 minute to station 1 and 4 minutes to station 2 . The second vehicle is located at a 5 -minute ride time from station 2.

Case 4: The first vehicle is located between station 1 and station 2, with a ride time of 4 minutes to station 1 and 1 minute to station 2 . The second vehicle is located at a 2 -minute ride time from station 2 .

As shown in Table 1, for the problem without time windows, there is no single best algorithm which suits all evaluated cases. For example, the IBR algorithm was one of the best in cases 1 and 4 , the SNN algorithm in cases 3 and 4, and the STN algorithm in cases 2 and 3. This shows that even in a very simple evaluation case, different situations require different algorithms. We therefore conclude that a mix of these algorithms may provide a more robust strategy, less sensitive to particular demand characteristics and vehicle distributions in the network.

As shown in Table 2, for the problem with time windows, there is also no single best algorithm which suits all evaluated cases-in every case, the number of passengers not served within their time windows (considered to be 10 minutes) reaches its minimum using either the IBR, SNN, or STN algorithm.

In previous articles [2-4], we have already shown that the combinations of algorithms show better performance for the problem without time windows. The preceding examples show that the use of combined methods of empty vehicle redistribution is also needed in the problem with time windows.

\section{COMPUTATIONAL EXPERIMENTS}

Evaluation of the algorithms for the empty vehicle redistribution problem with and without time windows is provided in this section. We evaluate the performance of different combinations of algorithms under different conditions.

Several combinations of algorithms introduced earlier in this article are studied and compared under two conditions. The first condition considers passenger waiting time windows. We aim 
to study here effects of passengers refusing the service after waiting more than the defined time threshold. Under the second condition, the redistribution algorithm takes these time windows into account, and it enables to refuse to serve some clients who cannot be served by the system within these waiting time limits.

The first main objective is to study the effect of adding client waiting time windows to the simulation (the implementation of the time windows to the problem setting in comparison with the problem without time windows). The second main objective is to study the effect of adding time-limited versions of the redistribution algorithms to take these time windows into account.

In the model implementation, we investigate the preceding conditions in the experiments with two different client arrival types as follows:

- Stationary Poisson point process,

- Based on the real taxi datasets.

We first introduce the case study followed by experiments with results discussions.

\subsection{Case Study}

The case study considered in this article is generated based on the real-world taxi floating car data from 1,500 taxis operating in the Stockholm, Sweden, region. Each taxi reports its GPS coordinates, timestamp, and status about its occupancy rate. Frequency of these reports is on average every 2 minutes. There is no special information about the true origin and destination of the trip. To infer them, the change in the status between two adjacent reports is used. The trip with client and $R$ reports $r_{1}, \ldots, r_{R}$ can be represented by binary vector like this: $(0,1,1, \ldots, 1,1,0)$, where 1 means that at the time of report $r_{i}$ the taxi is occupied and 0 otherwise. To represent the possible spatial space for true origin and destination, we consider them as the line or ordered vector of size 2 : $\left(r_{1}, r_{2}\right)$ for the origin and $\left(r_{R-1}, r_{R}\right)$ for the destination.

For the computational experiments, we consider all trips only with a client during morning peak hours (06:00-10:00) on April 1, 2016 (see Figure 2(a) for the spatial density of all trip origins in the Stockholm region). To simplify the network for the purpose of the computational experiments, we aggregate trips with origin and destination in a radius of $2 \mathrm{~km}$ in the surroundings of one of the 103 metro stations. Figure 2(b) and (c) show magnitudes of demand (number of trips) and destinations aggregated to the metro stations. The magnitude of the selected trips that originate and terminate across the metro stations constructs the OD matrix that can be used for a stationary Poisson point process as the trips themselves represent input for a real demand case with real call times. The distance matrix is constructed based on the spherical distance between all metro stations.

The expectancy of total number of clients arrived to all of the stations during the simulation period of 10,000 seconds is 1,451 .

\subsection{Combined Algorithms}

In combined algorithms, in every optimisation step, the system chooses the redistribution method and the number of available vehicles to redistribute. It is thus not necessary that the system will redistribute all vehicles available.

The selection of redistribution algorithm is based on random draws, given a predefined intensity $I_{i}$ for each algorithm, which is the number of times that algorithm is expected to be called per optimisation time interval. For example, let the IBR intensity be $I_{1}$, the SNN vehicle redistribution intensity be $I_{2}$, and the other algorithms' intensities be $I_{i}=0$. Then for each optimisation interval, there are $I=\left[I_{1}+I_{2}\right]$ random selections of redistribution algorithms with the probabilities $p_{1}=\frac{I_{1}}{I}$ and $p_{2}=\frac{I_{2}}{I}$. 


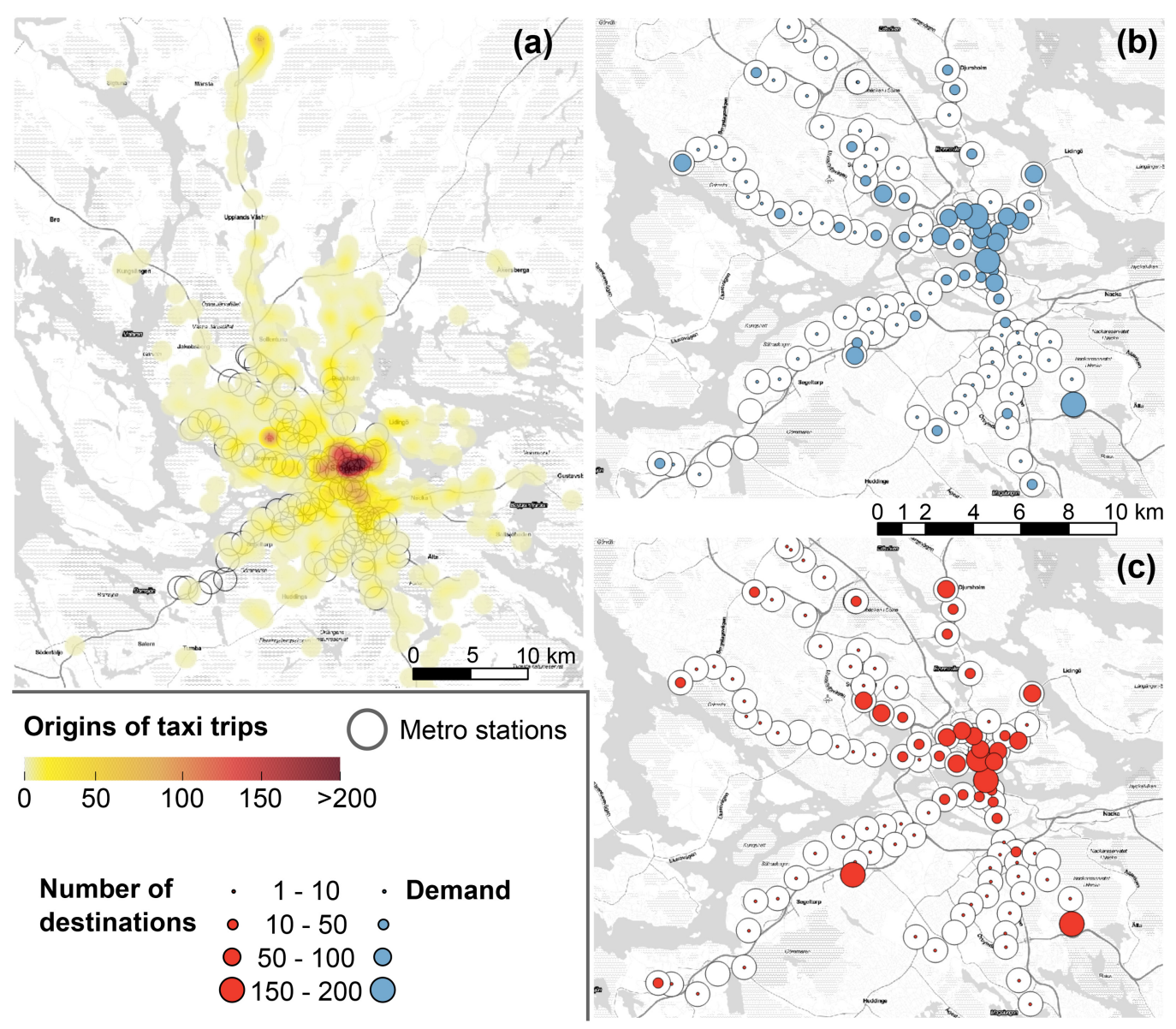

Fig. 2. Visualisation of the case study. (a) Spatial density of all trip origins before aggregation to metro stations. Demand aggregated across all metro stations (b) and destinations of the same trips (c).

Each module is independent and based on attributes of stations, vehicles and demands with parameters which can be set externally. The modules can be combined based on instantaneous conditions and/or time intervals, also controlled by parameters.

In the next section, the most relevant combinations for this article are presented. In every case, the results in the article are proposed as the solution of an optimisation subproblem on the algorithms' intensities.

The combined methods to compare in this article are as follows:

1.1 SNN+SDR: Simple Nearest Neighbours combined with Surplus/Deficit Redistribution

1.2 SNN+IBR: Simple Nearest Neighbours combined with Index Based Redistribution

1.3 SNN+IBRTL: Simple Nearest Neighbours combined with Index Based Redistribution Time Limited

2.1 HNN+SDR: Heuristic Nearest Neighbours combined with Surplus/Deficit Redistribution

$2.2 \mathrm{HNN+IBR}$ : Heuristic Nearest Neighbours combined with Index Based Redistribution

2.3 HNN+IBRTL: Heuristic Nearest Neighbours combined with Index Based Redistribution Time Limited 
3.1 HNNTL+SDR: Heuristic Nearest Neighbours Time Limited combined with Surplus/Deficit Redistribution

3.2 HNNTL+IBR: Heuristic Nearest Neighbours Time Limited combined with Index Based Redistribution

3.3 HNNTL+IBRTL: Heuristic Nearest Neighbours Time Limited combined with Index Based Redistribution Time Limited

4.1 STN+SDR: Send The Nearest combined with Surplus/Deficit Redistribution

4.2 STN+IBR: Send The Nearest combined with Index Based Redistribution

4.3 STN+IBRTL: Send The Nearest combined with Index Based Redistribution Time Limited

The evaluations of not combined reactive algorithms are provided in the appendix for every case study considered.

\subsection{Simulation on Poisson Client Arrivals}

The first simulation is made in supposition that the clients are arriving to stations based on Poisson distribution with constant rates for different stations. The modelling time is set to 10,000 seconds. The time window size in this case is 10 minutes, the number of vehicles is equal to 120 , and they are distributed uniformly at the stations when the simulation starts.

The purpose of the experiments provided in this article is to compare the system performance, defined as the objective functions (Section 3.2, optimisation problems 1, 2, and 3).

The number of necessary replications for simulation runs can be calculated from the desired size of the confidence intervals for the mean of the main outputs, as described in other work [11,9], where the following formula for the required number of replications $N$, given $m$ initial replications, is presented:

where

$$
N(m)=\left(\frac{S(m) t_{m-1,1-\alpha / 2}}{\bar{X}(m) \varepsilon}\right)^{2},
$$

- $N(m)$ is the number of replications required, given $m$ replications;

- $\bar{X}(m)$ is the estimate of the real mean $\mu$ from $m$ simulation runs (samples);

- $S(m)$ is the estimate of the real standard deviation $\sigma$ from $m$ simulation runs;

- $\alpha$ is level of significance;

- $\varepsilon$ is the allowable error percentage of the estimate $\bar{X}(m), \varepsilon=|\bar{X}(m)-\mu| / \mu$;

- $t_{m-1,1-\alpha / 2}$ is the critical value of the two-tailed $t$-distribution at a significance level $\alpha$, given $m-1$ degrees of freedom.

For every simulation case in this article, we calculate the necessary number of replications. The formula is applied to all objectives considered. Given $m=50$ initial replications, $\alpha=0.05$ and $\varepsilon=$ 0.1 , we obtain the required numbers of replications to obtain all objective parameters. For example, for the SNN+IBRTL combination, the formula shows that the required number of replications is equal to 84 . The number of replications does not exceed 100 for any algorithm combination and for any objective, and thus 100 simulations is used across all algorithm combinations.

5.3.1 The Comparison Between Different Redistribution Methods for the Problem with Time Windows. We provide the results of the numerical experiments for comparison of different redistribution algorithm combinations, for which we use the following abbreviations:

NotServ Number of not served clients

AvW Average waiting time within served clients, in minutes

MaxW Maximal waiting time within served clients, in minutes 
Table 3. Redistribution Methods Without TL for the Stockholm Network

\begin{tabular}{lcccccccc}
\hline & \multicolumn{4}{c}{ With Time Windows } & \multicolumn{3}{c}{ Without Time Windows } \\
\hline Combination & NotServ & AvW & MaxW & PrNotServ & Queue & Queue & AvW & MaxW \\
\hline 1.1 SNN+SDR & 129.44 & 6.2 & 10 & 8.9 & 35.22 & 115.6 & 11.4 & 27.7 \\
1.2 SNN+IBR & 118.36 & 6.2 & 10 & 8.1 & 31.76 & 48.9 & 9.1 & 26.5 \\
$2.1 \mathrm{HNN}+$ SDR & 238 & 5.8 & 9.99 & 16.4 & 30.78 & 111.6 & 11.5 & 28.9 \\
$2.2 \mathrm{HNN}+\mathrm{IBR}$ & 224.98 & 5.8 & 9.99 & 15.5 & 23.76 & 124.0 & 13.4 & 33.4 \\
4.1 STN+SDR & 59.82 & 2.4 & 9.91 & 4.1 & 11.84 & 16.9 & 3.1 & 27.5 \\
$4.2 \mathrm{STN}+\mathrm{IBR}$ & 55.5 & 2.9 & 9.95 & 3.8 & 9.58 & 9.1 & 3.455 & 18.2 \\
\hline
\end{tabular}

Table 4. Redistribution Methods for the Stockholm Network:

The Problem with Time Windows

\begin{tabular}{lcccc}
\hline Combination & NotServ & AvW & MaxW & PrNotServ \\
\hline 1.3 SNN+IBRTL & 80.14 & 5.9 & 9.99 & 5.5 \\
2.3 HNN+IBRTL & 112.24 & 6.2 & 9.99 & 7.7 \\
3.1 HNNTL+SDR & 110.12 & 6.2 & 9.99 & 7.6 \\
3.2 HNNTL+IBR & 85.92 & 6.1 & 9.99 & 5.9 \\
3.3 HNNTL+IBRTL & 97.98 & 6.0 & 9.98 & 6.7 \\
4.3 STN+IBRTL & 37.42 & 2.7 & 9.96 & 2.6 \\
\hline
\end{tabular}

PrNotServ Percentage of not served clients

Queue Queue length at the end of the simulation (Note: The value of queue length is the number of passengers not served yet at the end of simulation. All passengers remaining at the end of the simulation may be served later within their time windows.)

Table 3 provides the first results. We investigate two cases here. First, clients with a time window (columns 2-6 in Table 3) where clients are not served within 10 minutes will quit the system. Second, clients are waiting as long as they are served and never leave simulation until they are served (last three columns in Table 3).

Not surprisingly, the results in Table 3 show that in the terms of the average waiting time, the algorithms applied to the case with the time window are better solutions. Nevertheless, this difference is achieved by the high number of not served passengers. In different problems occurring in transportation, the utility function of clients contains not only the waiting time but also if the waiting was successful. Thus, when choosing between strategies with close average waiting times, the number of served passengers and the queue length have to be taken into account. The combination with the STN algorithm can in this case help improve the number of served clients and average waiting times within served clients.

5.3.2 Effects of Considering Client Waiting Time Windows in Redistribution Algorithms. In the case with client time windows, the application of the algorithms with abbreviations TL (time limited) introduced in Section 4.2 is possible.

Comparing Table 3 and Table 4 reveals that the combinations with time limited variations of algorithms outperform combinations without TL in terms of number of clients served within the time window. Nevertheless, in terms of average waiting times of the clients served, there are no significant differences. The maximal waiting times obtained for all algorithms considered for the problem with time windows are close to the size of the time window. 
Table 5. Redistribution Methods for the Stockholm Network, Small Demand

\begin{tabular}{lcccccccc}
\hline & \multicolumn{4}{c}{ With Time Windows } & \multicolumn{4}{c}{ Without Time Windows } \\
\hline Combination & NotServ & AvW & MaxW & PrNotServ & Queue & Queue & AvW & MaxW \\
\hline 1.1 SNN+SDR & 1.72 & 1.33 & 7.67 & 0.23 & 1.94 & 1.24 & 1.14 & 7.85 \\
1.2 SNN+IBR & 0.02 & 0.68 & 6.32 & 0.003 & 0.02 & 0.14 & 0.67 & 6.18 \\
1.3 SNN+IBRTL & 0.14 & 0.67 & 6.22 & 0.02 & 0.12 & - & - & - \\
2.1 HNN+SDR & 2.14 & 1.31 & 7.87 & 0.29 & 1.98 & 1.18 & 1.21 & 7.96 \\
2.2 HNN+IBR & 0.22 & 0.65 & 6.18 & 0.03 & 0.2 & 0.16 & 0.76 & 6.64 \\
2.3 HNN+IBRTL & 0.14 & 0.71 & 6.23 & 0.02 & 0.18 & - & - & - \\
3.1 HNNTL+SDR & 1.66 & 1.15 & 7.43 & 0.23 & 2.2 & - & - & - \\
3.2 HNNTL+IBR & 0.06 & 0.66 & 6.04 & 0.008 & 0.12 & - & - & - \\
3.3 HNNTL+IBRTL & 0.16 & 0.79 & 6.75 & 0.02 & 0.06 & - & - & - \\
4.1 STN+SDR & 0.46 & 0.66 & 7.52 & 0.06 & 2.04 & 0.62 & 0.73 & 8.42 \\
4.2 STN+IBR & 0.06 & 0.40 & 6.43 & 0.008 & 0.18 & 0.2 & 0.45 & 6.01 \\
4.3 STN+IBRTL & 0.02 & 0.43 & 6.02 & 0.003 & 0.12 & - & - & - \\
\hline
\end{tabular}

The results also show that in combination, it seems to be sufficient to use only one of the heuristical algorithms which takes into account the clients' time windows. For example, when the reactive method in the simulation is HNNTL, the change of the proactive one from IBR to IBRTL does not improve the overall performance. The most robust combination for the case with time windows in the redistribution algorithm is STN+IBRTL and, in comparison to STN+IBT, the considering time limits in redistribution helps decrease most attributes. The most significant drop is in the number of not served clients from 55.5 to 37.42 , which is about $32 \%$.

5.3.3 Effects of Demand. To show the robustness of the empty vehicle redistribution methods proposed, it is important to provide the performance at different demand levels. If in the scenario of high demand level serving all the clients is not possible, less demand can theoretically be served completely or close to completely. The evaluation of the same algorithms applied to less demand (by considering $50 \%$ of the original demand) is shown in Table 5.

Table 5 is organised the same way as Table 3 . The $\langle<->>$ in the table means that these data are not available, because TL versions of algorithms cannot be used for that case. The simulation results show that for the case with time windows, the combinations SNN+IBRTL, HNNTL+IBR, and STN+IBRTL seem to be promising in terms of number of clients served within the time window. For the problem without time windows, the combination STN+IBR shows the best results in terms of both average and maximal waiting times. Here, the difference between STN-based algorithms and SNN ones is not so significant for both problems, with time windows and without time windows.

The maximal passenger waiting times in the problem with time windows show, in contrast to a full demand scenario, smaller values than the size of time window.

The difference in the results of the simulations with and without clients' time windows is not significant for identical methods. For example, when applying the STN+IBR method, the maximal waiting time within served clients is 6.43 for the simulation with time windows and 6.01 for the case without time windows; whenever the average times are 0.40 and 0.45 , respectively, the total number of not served passengers is $0.06+0.18=0.24$ (the number of passengers that have quit the system plus the number of passengers remaining at the end of the simulation) in the case with time windows and 0.2 in the case without time windows. Thus, the overall performance level is of the same order. 


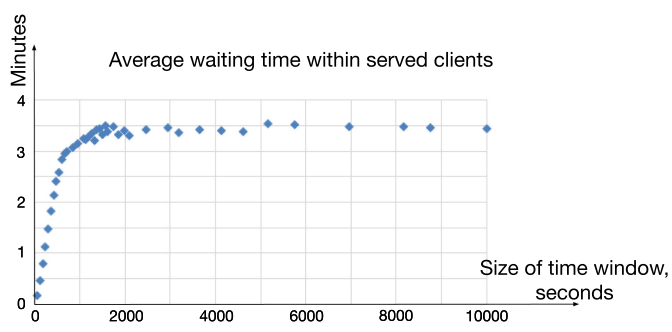

(a)

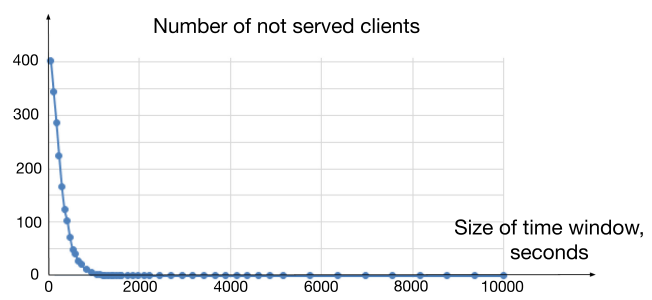

(b)

Fig. 3. (a) Time window influence to average waiting time for served clients. (b) Time window influence to number of not served clients.

For the case with time windows, the method STN+IBRTL shows the best results in overall performance, showing the best results in terms of maximal waiting time and number of not served passengers and one of the best results in terms of average waiting time and queue length.

5.3.4 Influence of time Window Size on System Performance. To provide a reasoning for the stated level of service in autonomous taxi systems, the analysis of the time window size is provided. Considering that the time window is equal to the time limit in TL versions of algorithms, we provide the performance analysis for the best of the cases in terms of clients served for the full demand scenario, STN+IBRTL. The aim is to show the number of clients served, and the average and maximal waiting times depending on time window size.

The results of the simulation show shape with negative gradient in terms of number of clients not served within their time window constraints (Figure 3(a)). In terms of average waiting time for served clients, Figure 3(b) shows that until reaching the critical time window size value, the average waiting time for served clients will be oscillating around 3.5 minutes (whenever the maximal waiting times can be significantly big and achieve the current time window size).

\subsection{Simulation on Real Demand}

The third simulation uses real demand data, using the taxi departure times of every trip provided by the Stockholm taxi dataset. Nevertheless, the IBR and IBRTL algorithms are assuming the Poisson nature of predicted future arrivals. In this scenario, the comparison between different redistribution algorithms in Table 6 shows that in terms of number of not served clients, the combination $\mathrm{STN}+\mathrm{IBR}$ outperforms the other combined algorithms. For the problem without time window constraints, this combination significantly outperforms the others in terms of maximal waiting time. In the problem without time windows, the best performance in terms of average waiting time was obtained by combination with STN, and especially the STN+SDR method.

The strategies HNN+SDR and HNN+IBR show the worst performances in terms of the number of not served clients for the case with time windows. Nevertheless, the HNN+IBRTL combination shows better results than SNN+IBRTL. This observation can be explained by an appeal to the formulation of the algorithms: in the HNN algorithm, the vehicle is to be sent to the maximal (at the moment of the nearest vehicle arrival) waiting client, without taking into account the fact that the client could already quit the system because the client has to wait more than 10 minutes.

\section{CONCLUSION}

In this article, we investigate empty vehicle redistribution algorithms for PRT or autonomous taxi services, primarily from a passenger service perspective. Using a simple case, we first evaluate 
Table 6. Comparison Between Different Redistribution Methods for the Stockholm Network with Real Demand Data

\begin{tabular}{lcccccccc}
\hline & \multicolumn{4}{c}{ With Time Windows } & \multicolumn{3}{c}{ Without Time Windows } \\
\hline Combination & NotServ & AvW & MaxW & PrNotServ & Queue & Queue & AvW & MaxW \\
\hline 1.1 SNN+SDR & 118 & 5.47 & 10 & 8.16 & 143 & 249 & 6.03 & 25.1 \\
1.2 SNN+IBR & 106 & 5.27 & 10 & 7.30 & 125 & 284 & 7.99 & 36.66 \\
1.3 SNN+IBRTL & 81 & 4.92 & 9.97 & 5.55 & 122 & - & - & - \\
$2.1 \mathrm{HNN+SDR}$ & 240 & 2.95 & 9.99 & 16.56 & 132 & 358 & 7.09 & 30.04 \\
2.2 HNN+IBR & 263 & 3.74 & 10 & 18.16 & 137 & 286 & 5.35 & 33.84 \\
2.3 HNN+IBRTL & 56 & 3.84 & 10 & 3.88 & 116 & - & - & - \\
3.1 HNNTL+SDR & 83 & 3.49 & 9.96 & 5.75 & 125 & - & - & - \\
3.2 HNNTL+IBR & 81 & 4.33 & 9.99 & 5.59 & 124 & - & - & - \\
3.3 HNNTL+IBRTL & 76 & 3.91 & 9.98 & 5.22 & 123 & - & - & - \\
4.1 STN+SDR & 48 & 1.91 & 9.95 & 3.30 & 57 & 68 & 2.43 & 31.83 \\
4.2 STN+IBR & 39 & 1.98 & 9.93 & 2.69 & 75 & 86 & 2.97 & 21.40 \\
4.3 STN+IBRTL & 43 & 2.62 & 9.97 & 2.93 & 84 & - & - & - \\
\hline
\end{tabular}

three algorithms for four different cases of initial vehicle locations and demonstrate that none of the algorithms is superior in all possible cases.

Using computational experiments on the Stockholm network and real taxi dataset for demand, we investigated the effects of two main conditions. The first condition is the time window concept that adds the constraints to the passenger waiting times. The second condition tested is the concept of incorporating the passenger waiting time limit in the redistribution algorithms, and thus the system can reject demands that will be not served. Considering passenger waiting time windows allows to model them in a more realistic way as in the real world to one willing to wait infinite time for service. By the second condition, we involved this time limit consideration to the redistribution algorithms which helps improve almost all attributes we studied. Under this condition, new IBRTL and HNNTL algorithms that improve on existing empty vehicle redistribution algorithms are presented. The number of not served clients can be lowered about $32 \%$ just by adaptive redistribution towards waiting clients' times.

The results presented in this article show that the IBR-based methods perform well for provided demand, especially in combination with existing nearest neighbours methods such as SNN and STN. The results on the Stockholm network show that the STN+IBRTL redistribution method is one of the most robust and best performing for the problem with time windows.

The implementation of proposed algorithms to the real demand case shows that STN-based methods of empty vehicle redistribution show the best results in terms of the number of clients served within their constraints, and the STN+IBR method shows the significant improvement of maximal waiting time for the problem without time windows.

The algorithms described in the article assumed that information about departure stations of the clients was known at the time of vehicle redistribution, with the destination station of the client being assigned when it boards the vehicle. Incorporating heuristics on client OD demand may improve our methods further and provide the sufficient model with the ride sharing.

In this work, we focus purely on the passenger perspective, ignoring the cost of empty vehicle runs to the operator. In previous work [2], it is shown that using the SNN+IBR strategy for the problem without time windows, the good performance in terms of passenger waiting times and queue lengths comes at the cost of a large number of empty vehicle runs.

In the future, we plan to include stochastic client waiting time constraints, as well as charging constraints. 


\section{APPENDICES}

\section{A USED NOTATIONS}

$t$ Modelling time in the model

$G=G(V, E)$ Directed graph of the city

$v \in V$ Vertex of the city graph

$e \in E$ Arc of the city graph

init $_{e}$ Initial vertex of arc $e$

ter $e_{e}$ Terminal vertex of arc $e$

time $_{e}$ Time to cross arc $e$ at time moment $t$

$p \in P$ Client (passenger group, travelling together)

$N_{p}$ Number of passengers for client $p$

$v_{p}^{o} \in V$ Origin of client $p$

$v_{p}^{d} \in V$ Destination of client $p$

$t_{p}^{0}$ Minimal accepted boarding time of client $p$

$t_{p}^{1}$ Maximal accepted boarding time of client $p$

$T W=t_{p}^{1}-t_{p}^{0}$ Time window size of client $p$

$t_{p}^{a p p}$ Taxi calling time of client $p$

$F_{p}$ If flag of the serving of client $p$ is within its utility constraints

$t_{p}^{\text {dep }}$ Real departure time of client $p$

$u_{p}\left(t_{p}^{d e p}-t_{p}^{0}\right)$ Utility of client $p$

$P_{s, a r r>0}\left(t_{1}, t_{1}+\tau\right)$ Probability of at least one client arrival to station $s$ during the time period $\left(t_{1}, t_{1}+\tau\right)$

$v_{s}^{\text {nearest }}$ Nearest available vehicle to station $s \in V$

$P S_{s}$ Client surplus on station $s$

$I_{s}$ Index of station $s$

$T_{s}$ Maximal waiting time among the clients at station $s$

$T_{s, X}$ Time expectancy of $X$ clients arriving to station $s$

$T_{s}^{m}$ Maximal waiting time within client at station $s$ at the moment of the nearest vehicle arriving to station $s$

$C S_{s}$ Client surplus of station $s$

BA Basic Allocation

IBR Index Based Redistribution

HNN Heuristic Nearest Neighbours

SDR Surplus/Deficit vehicle Redistribution

SNN Simple Nearest Neighbours

STN Send The Nearest

IBRTL Index Based Redistribution Time Limited

HNNTL Heuristic Nearest Neighbours Time Limited

\section{B EVALUATION OF THE COMBINED EMPTY VEHICLE REDISTRIBUTION ALGORITHMS FOR THE STOCKHOLM CASE STUDY: FULL TABLES}

The methods to compare are as follows:

0.0 BA. Basic Allocation. This means no redistribution of empty vehicles between the stations, baseline for comparison with the other strategies

1.0 SNN. Simple Nearest Neighbours 
1.1 SNN+SDR. Simple Nearest Neighbours combined with Surplus/Deficit vehicle Redistribution

1.2 SNN+IBR. Simple Nearest Neighbours combined with Index Based Redistribution

1.3 SNN+IBRTL. Simple Nearest Neighbours combined with Index Based Redistribution Time Limited

2.0 HNN. Heuristic Nearest Neighbours

2.1 HNN+SDR. Heuristic Nearest Neighbours combined with Surplus/Deficit vehicle Redistribution

2.2 HNN+IBR. Heuristic Nearest Neighbours combined with Index Based Redistribution

2.3 HNN+IBRTL. Heuristic Nearest Neighbours combined with Index Based Redistribution Time Limited

3.0 HNNTL. Heuristic Nearest Neighbours Time Limited

3.1 HNNTL+SDR. Heuristic Nearest Neighbours Time Limited combined with Surplus/Deficit vehicle Redistribution

3.2 HNNTL+IBR. Heuristic Nearest Neighbours Time Limited combined with Index Based Redistribution

3.3 HNNTL+IBRTL. Heuristic Nearest Neighbours Time Limited combined with Index Based Redistribution Time Limited

4.0 STN. Send The Nearest

4.1 STN+SDR. Send The Nearest combined with Surplus/Deficit vehicle Redistribution

4.2 STN+IBR. Send The Nearest combined with Index Based Redistribution

4.3 STN+IBRTL. Send The Nearest combined with Index Based Redistribution Time Limited

In the tables, we use the following abbreviations:

NotServ Number of not served clients.

AvW Average waiting time within served clients, in minutes.

MaxW Maximal waiting time within served clients, in minutes.

PrNotServ Percentage of not served clients.

Queue Queue length at the end of the simulation. The value of queue length for the simulation is the number of passengers not served yet at the end of simulation. All passengersstaying at the end of the simulation may be served later within their time windows.

The tables below are organised as follows. The first column is the type of combination presented in the row. We investigate two cases here. First, clients with time windows (columns 2-6) where clients not served within 10 minutes will quit the system. Second, clients are waiting as long as they are served and never leave simulation until they are served (last 3 columns). The $<<->>$ in the table means that this data is not available because it uses TL versions of algorithms. 
Table 7. Comparison Between Different Redistribution Methods for the Stockholm Network: Full Table

\begin{tabular}{lcccccccc}
\hline & \multicolumn{4}{c}{ With Time Windows } & \multicolumn{3}{c}{ Without Time Windows } \\
\hline Strategy & NotServ & AvW & MaxW & PrNotServ & Queue & Queue & AvW & MaxW \\
\hline 0.0 BA & 1340 & 7.9 & 10 & 92.4 & 64.74 & 1426 & 28.6 & 84.9 \\
$1.0 \mathrm{SNN}$ & 140.88 & 6.4 & 10 & 9.73 & 38.58 & 76.5 & 8.8 & 24.7 \\
$1.1 \mathrm{SNN}+$ SDR & 129.44 & 6.2 & 10 & 8.9 & 35.22 & 115.6 & 11.4 & 27.7 \\
$1.2 \mathrm{SNN}+\mathrm{IBR}$ & 118.36 & 6.2 & 10 & 8.1 & 31.76 & 48.9 & 9.1 & 26.5 \\
$1.3 \mathrm{SNN}+\mathrm{IBRTL}$ & 80.14 & 5.9 & 9.99 & 5.5 & 20.9 & - & - & - \\
2.0 HNN & 289.88 & 6.0 & 9.99 & 19.9 & 38.56 & 112.4 & 11.9 & 29.5 \\
2.1 HNN+SDR & 238 & 5.8 & 9.99 & 16.4 & 30.78 & 111.6 & 11.5 & 28.9 \\
2.2 HNN+IBR & 224.98 & 5.8 & 9.99 & 15.5 & 23.76 & 124.0 & 13.4 & 33.4 \\
2.3 HNN+IBRTL & 112.24 & 6.2 & 9.99 & 7.7 & 18.8 & - & - & - \\
3.0 HNNTL & 120.68 & 6.2 & 9.98 & 8.3 & 33.86 & - & - & - \\
3.1 HNNTL+SDR & 110.12 & 6.2 & 9.99 & 7.6 & 40.28 & - & - & - \\
3.2 HNNTL+IBR & 85.92 & 6.1 & 9.99 & 5.9 & 25.08 & - & - & - \\
3.3 HNNTL+IBRTL & 97.98 & 6.0 & 9.98 & 6.7 & 20.64 & - & - & - \\
4.0 STN & 65.50 & 2.5 & 9.95 & 4.5 & 11.82 & 17.8 & 3.3 & 28.8 \\
4.1 STN+SDR & 59.82 & 2.4 & 9.91 & 4.1 & 11.84 & 16.9 & 3.1 & 27.5 \\
4.2 STN+IBR & 55.5 & 2.9 & 9.95 & 3.8 & 9.58 & 9.1 & 3.455 & 18.2 \\
4.3 STN+IBRTL & 37.42 & 2.7 & 9.96 & 2.6 & 8.14 & - & - & - \\
\hline
\end{tabular}

Table 8. Comparison Between Different Redistribution Methods for the Stockholm Network, Small Demand: Full Table

\begin{tabular}{lcccccccc}
\hline & \multicolumn{4}{c}{ With Time Windows } & \multicolumn{3}{c}{ Without Time Windows } \\
\hline Strategy & NotServ & AvW & MaxW & PrNotServ & Queue & Queue & AvW & MaxW \\
\hline 0.0 BA & 587.46 & 8.22 & 9.99 & 80.97 & 23.84 & 667 & 35.7 & 99.5 \\
$1.0 \mathrm{SNN}$ & 1.2 & 1.14 & 7.50 & 0.16 & 2.04 & 0.8 & 1.26 & 7.93 \\
$1.1 \mathrm{SNN}+$ SDR & 1.72 & 1.33 & 7.67 & 0.23 & 1.94 & 1.24 & 1.14 & 7.85 \\
$1.2 \mathrm{SNN}+\mathrm{IBR}$ & 0.02 & 0.68 & 6.32 & 0.003 & 0.02 & 0.14 & 0.67 & 6.18 \\
$1.3 \mathrm{SNN}+\mathrm{IBRTL}$ & 0.14 & 0.67 & 6.22 & 0.002 & 0.12 & - & - & - \\
$2.0 \mathrm{HNN}$ & 3.34 & 1.33 & 7.85 & 0.46 & 2.34 & 0.86 & 1.22 & 8.10 \\
$2.1 \mathrm{HNN}+$ SDR & 2.14 & 1.31 & 7.87 & 0.29 & 1.98 & 1.18 & 1.21 & 7.96 \\
2.2 HNN+IBR & 0.22 & 0.65 & 6.18 & 0.03 & 0.2 & 0.16 & 0.76 & 6.64 \\
$2.3 \mathrm{HNN+IBRTL}$ & 0.14 & 0.71 & 6.23 & 0.02 & 0.18 & - & - & - \\
3.0 HNNTL & 1.38 & 1.23 & 7.59 & 0.19 & 1.4 & - & - & - \\
3.1 HNNTL+SDR & 1.66 & 1.15 & 7.43 & 0.23 & 2.2 & - & - & - \\
3.2 HNNTL+IBR & 0.06 & 0.66 & 6.04 & 0.008 & 0.12 & - & - & - \\
3.3 HNNTL+IBRTL & 0.16 & 0.79 & 6.75 & 0.02 & 0.06 & - & - & - \\
4.0 STN & 0.7 & 0.67 & 7.90 & 0.1 & 0.34 & 0.34 & 0.79 & 8.73 \\
4.1 STN+SDR & 0.46 & 0.66 & 7.52 & 0.06 & 2.04 & 0.62 & 0.73 & 8.42 \\
4.2 STN+IBR & 0.06 & 0.40 & 6.43 & 0.008 & 0.18 & 0.2 & 0.45 & 6.01 \\
4.3 STN+IBRTL & 0.02 & 0.43 & 6.02 & 0.003 & 0.12 & - & - & - \\
\hline
\end{tabular}


Table 9. Comparison Between Different Redistribution Methods for the Stockholm Network with Real Demand Data: Full Table

\begin{tabular}{lcccccccc}
\hline & \multicolumn{4}{c}{ With Time Windows } & \multicolumn{3}{c}{ Without Time Windows } \\
\hline Strategy & NotServ & AvW & MaxW & PrNotServ & Queue & Queue & AvW & MaxW \\
\hline 0.0 BA & 1252 & 8.55 & 10 & 86.3 & 146 & 1444 & 21.16 & 75.99 \\
1.0 SNN & 121 & 5.54 & 10 & 8.36 & 138 & 297 & 5.94 & 29.4 \\
1.1 SNN+SDR & 118 & 5.47 & 10 & 8.16 & 143 & 249 & 6.03 & 25.1 \\
1.2 SNN+IBR & 106 & 5.27 & 10 & 7.30 & 125 & 284 & 7.99 & 36.66 \\
1.3 SNN+IBRTL & 81 & 4.92 & 9.97 & 5.55 & 122 & - & - & - \\
2.0 HNN & 170 & 2.10 & 9.99 & 11.70 & 131 & 297 & 5.14 & 33.6 \\
2.1 HNN+SDR & 240 & 2.95 & 9.99 & 16.56 & 132 & 358 & 7.09 & 30.04 \\
2.2 HNN+IBR & 263 & 3.74 & 10 & 18.16 & 137 & 286 & 5.35 & 33.84 \\
2.3 HNN+IBRTL & 56 & 3.84 & 10 & 3.88 & 116 & - & - & - \\
3.0 HNNTL & 89 & 4.75 & 10 & 6.16 & 126 & - & - & - \\
3.1 HNNTL+SDR & 83 & 3.49 & 9.96 & 5.75 & 125 & - & - & - \\
3.2 HNNTL+IBR & 81 & 4.33 & 9.99 & 5.59 & 124 & - & - & - \\
3.3 HNNTL+IBRTL & 76 & 3.91 & 9.98 & 5.22 & 123 & - & - & - \\
4.0 STN & 42 & 2.05 & 9.87 & 2.86 & 63 & 82 & 2.33 & 32.80 \\
4.1 STN+SDR & 48 & 1.91 & 9.95 & 3.30 & 57 & 68 & 2.43 & 31.83 \\
4.2 STN+IBR & 39 & 1.98 & 9.93 & 2.69 & 75 & 86 & 2.97 & 21.40 \\
4.3 STN+IBRTL & 43 & 2.62 & 9.97 & 2.93 & 84 & - & - & - \\
\hline
\end{tabular}

\section{REFERENCES}

[1] Ingmar Andréasson. 2003. Reallocation of empty personal rapid transit vehicles en route. Transportation Research Record 1838 (2003), 36-41.

[2] Tatiana Babicheva, Wilco Burghout, Ingmar Andréasson, and Nadege Faul. 2018. Empty vehicle redistribution and fleet-size in autonomous taxi systems. IET Intelligent Transport Systems 13, 4 (2018), 677-682.

[3] Tatiana Babicheva, Wilco Burghout, Ingmar Andréasson, and Nadege Faul. 2018. The matching problem of empty vehicle redistribution in autonomous taxi systems. Procedia Computer Science 130 (2018), 119-125.

[4] Tatiana Babicheva, Wilco Burghout, Ingmar Andréasson, and Nadege Faul. 2018. The matching problem of empty vehicle redistribution in autonomous taxi systems. Procedia Computer Science 130 (2018), 119-125.

[5] Gary S. Becker. 1965. A theory of the allocation of time. Economic fournal 75, 299 (1965), 493-517.

[6] Michael G. H. Bell and K. I. Wong. 2005. A rolling horizon approach to the optimal dispatching of taxis. In Proceedings of the 16th International Symposium on Transportation and Traffic Theory: Flow, Dynamics, and Human Interaction. 629-648.

[7] Maria Börjesson and Jonas Eliasson. 2014. Experiences from the Swedish value of time study. Transportation Research Part A: Policy and Practice 59 (2014), 144-158.

[8] Leo Breiman. 1963. The Poisson tendency in traffic distribution. Annals of Mathematical Statistics 34, 1 (1963), $308-311$.

[9] Wilco Burghout. 2004. A Note on the Number of Replication Runs in Stochastic Traffic Simulation Models. Unpublished Report. Centre for Traffic Research, Stockholm, Sweden.

[10] Wilco Burghout, Pierre Jean Rigole, and Ingmar Andréasson. 2015. Impacts of shared autonomous taxis in a metropolitan area. In Proceedings of the 94th Annual Meeting of the Transportation Research Board.

[11] Nicolas Chiabaut and Christine Buisson. 2009. Replications in stochastic traffic flow models: Incremental method to determine sufficient number of runs. In Traffic and Granular Flow '07. Springer, 35-44.

[12] Allan C. DeSerpa. 1971. A theory of the economics of time. Economic fournal 81, 324 (1971), 828-846.

[13] Martin Desrochers, Jacques Desrosiers, and Marius Solomon. 1992. A new optimization algorithm for the vehicle routing problem with time windows. Operations Research 40, 2 (1992), 342-354.

[14] Alan W. Evans. 1972. On the theory of the valuation and allocation of time. Scottish fournal of Political Economy 19, 1 (1972), 1-17. 
[15] Daniel J. Fagnant and Kara M. Kockelman. 2018. Dynamic ride-sharing and fleet sizing for a system of shared autonomous vehicles in Austin, Texas. Transportation 45 (2018), 143-158.

[16] Ezzeddine Fatnassi, Olfa Chebbi, and Jouhaina Chaouachi. 2017. Dealing with the empty vehicle movements in personal rapid transit system with batteries constraints in a dynamic context. Fournal of Advanced Transportation 2017 (2017), Article 8512728.

[17] Richard Gilbert and Anthony Perl. 2007. Grid-connected vehicles as the core of future land-based transport systems. Energy Policy 35, 5 (2007), 3053-3060.

[18] Kyle Guan, Joseph Kakande, and Junho Cho. 2016. On deploying encryption solutions to provide secure transport-asa-service (TaaS) in core and metro networks. In Proceedings of the 42 nd European Conference on Optical Communication (ECOC'16). 1-3.

[19] Robert Hendershot. 1975. Morgantown Personal Rapid Transit System, System Operation Description Manual. Technical Report. M-PRT-1-1. Boeing Aerospace.

[20] Lars Hultkrantz and Reza Mortazavi. 2001. Anomalies in the value of travel-time changes. fournal of Transport Economics and Policy 35, 2 (2001), 285-299.

[21] Sergio R. Jara-Díaz. 2008. Allocation and valuation of travel time savings. Handbook of Transport Modelling 2 (2008), 363-379.

[22] Shenggong Ji. 2019. A deep reinforcement learning-enabled dynamic redeployment system for mobile ambulances. In Proceedings of the ACM on Interactive, Mobile, Wearable, and Ubiquitous Technologies, Vol. 3.1 (2019), 15. ACM, New York, NY.

[23] Alvina Kek, Ruey Cheu, and Miaw Chor. 2006. Relocation simulation model for multiple-station shared-use vehicle systems. Transportation Research Record 1986 (2006), 81-88.

[24] John D. Lees-Miller. 2016. Minimising average passenger waiting time in personal rapid transit systems. Annals of Operations Research 236, 2 (2016), 405-424.

[25] Yexin Li, Zheng Yu, and Yang Qiang. 2019. Efficient and effective express via contextual cooperative reinforcement learning. In Proceedings of the 25th ACM SIGKDD International Conference on Knowledge Discovery and Data Mining. ACM, New York, NY.

[26] Thomas E. Lisco. 1968. Value of Commuters Travel Time: A Study in Urban Transportation. Technical Report. Highway Research Record.

[27] Phathinan Thaithatkul, Toru Seo, Takahiko Kusakabe, and Yasuo Asakura. 2016. Simulation approach for investigating dynamics of passenger matching problem in smart ridesharing system. In Proceedings of the International Symposium of Transport Simulation and the International Workshop on Traffic Data Collection and Its Standardisation.

[28] William G. Waters. 1994. The value of travel time savings and the link with income: Implications for public project evaluation. International fournal of Transport Economics 21, 3 (1994), 243-253.

[29] Yale Z. Wong, David A. Hensher, and Corinne Mulley. 2017. Emerging transport technologies and the modal efficiency framework: A case for mobility as a service (MaaS). In Proceedings of the 15th International Conference on Competition and Ownership in Land Passenger Transport.

[30] Zhe Xu. 2018. Large-scale order dispatch in on-demand ride-hailing platforms: A learning and planning approach.. In Proceedings of the 24th ACM SIGKDD International Conference on Knowledge Discovery and Data Mining. ACM, New York, NY.

Received June 2019; revised June 2020; accepted August 2020 